\title{
Glial cell line-derived neurotrophic factor (GDNF) improves spatial learning in aged Fischer 344 rats
}

\author{
MARY ANN PELLEYMOUNTER, MARY JANE CULLEN, MARY BETH BAKER, and DENIS HEALY \\ Amgen, Inc., Thousand Oaks, California
}

\begin{abstract}
Glial cell line-derived neurotrophic factor (GDNF) increases choline acetyltransferase (ChAT) activity in the septohippocampal system of aged rats. Since the septohippocampal system has been implicated as a neurobiological substrate for spatial learning, the effects of GDNF were tested in aged, spatial learning-impaired Fisher 344 rats. Aged rats were characterized as spatial learning "impaired" or "unimpaired" using several indices of performance in the Morris water maze, and they were then given GDNF (100- $\mu$ g bolus; icv) or phosphate-buffered saline (PBS). Six days after the GDNF or PBS injection, rats returned to water maze training, which continued until a spatial bias criterion was met. Immediately after meeting the criterion, rats were tested in a cue learning version of the task. GDNF significantly improved spatial learning in aged impaired rats, but not aged unimpaired or young rats. It did not alter cue learning rate for any group, nor did it alter swim speed. These data suggest that GDNF may support the integrity of neurons in the septum and hippocampus of aged rats.
\end{abstract}

Glial cell line-derived neurotrophic factor (GDNF) is best known for its effects on mesencephalic dopaminergic neurons. It promotes the survival and differentiation of mesencephalic dopaminergic neurons in vitro (Lin, Doherty, Lile, Bektesh, \& Collins, 1993). Furthermore, it partially protects and reverses neurotoxin-induced dopaminergic neuron degeneration in adult rats and nonhuman primates (Beck et al., 1995; Hoffer et al., 1994; Kearns \& Gash, 1995; Sauer, Rosenblad, \& Bjorklund, 1995; Tomac et al., 1995). Recent data have suggested that GDNF can protect neurons with other neurochemical phentoypes as well. For example, GDNF has been shown to promote the survival of developing sensory, autonomic, and motor neurons (Henderson et al., 1994; Oppenheim et al., 1995; Yan, Matheson, \& Lopez, 1995), along with protecting these neurons from degeneration after injury in adults (Lin et al., 1993). GDNF has also been shown to protect cholinergic septohippocampal neurons from degeneration after a fimbria/fornix transection (Williams, Inouye, Cummins, \& Pelleymounter, 1996), as well as dramatically increasing septal, hippocampal, cortical, and striatal choline acetyltransferase (ChAT) activity in aged and young Fischer 344 rats (Lapchak, Miller, \& Jiao, 1997). The fact that GDNF could protect septohippocampal neurons suggested that GDNF might be effective in cognitive tasks such as the Morris water maze, which is dependent on the integrity of this pathway (Devan, Goad, \& Petri, 1996; Eichenbaum, Stewart, \& Morris, 1990). We tested the hypothesis that GDNF could affect

Correspondence should be addressed to $M$. A. Pelleymounter, Department of Neuroscience, Neurocrine Biosciences, 10555 Science Center Dr., San Diego, CA 92121 (e-mail: mpelleymounter@neurocrine. com). cognition by assessing its effects on spatial learning in young and aged Fischer 344 rats.

\section{METHOD}

\section{Subjects}

Thirty aged Fischer 344 male rats (23-25 months old) and 30 young male Fischer 344 rats (3-4 months old) were used as subjects in the study. All animals were acquired from Harlan SpragueDawley and housed individually in the Amgen vivarium up to and during the test periods. The rats were on a 12:12-h light:dark cycle (lights on 7 a.m., lights off 7 p.m.) and were allowed food and water ad libitum. All behavioral testing was conducted between 9 a.m. and 3 p.m.

\section{Apparatus}

The Morris water maze was $1.8 \mathrm{~m}$ in diameter, with a depth of $61 \mathrm{~cm}$. Wall hangings and shelves provided extramaze cues. In the place version of the task, the escape platform was submerged $1 \mathrm{~cm}$ below the surface of tepid $\left(28^{\circ} \mathrm{C}\right)$ water made opaque by the addition of powdered milk $(0.9 \mathrm{~kg})$. In the cue version of the task, the escape platform was black and extended $1 \mathrm{~cm}$ above the surface of the water, which was $30 \mathrm{~cm}$ deep. A closed-circuit TV camera was suspended above the midpoint of the maze. The camera was part of a computerized tracking system developed by San Diego Instruments that monitored the rats' swim distance and escape latency.

\section{Procedure}

For each trial, the rats were placed into the maze and allowed to search for the hidden escape platform for up to $90 \mathrm{sec}$. If they had not located the platform in this time period, the experimenter guided them to the platform. After reaching the platform, the rats were allowed to remain on it for $15 \mathrm{sec}$, at which point they were given an intertrial interval of $60 \mathrm{sec}$ in a dry cage. The rats were placed into the maze at one of four cardinal start points for each trial, with start points varying from trial to trial. The rats were given four trials each day. Both latency and distance to reach the escape platform were recorded for each trial.

The aged rats were identified as "impaired" or "unimpaired" on the basis of their performance in 32 initial place training trials, 
where the 32 nd trial served as a probe trial. During a probe trial, the escape platform was removed, and certain aspects of the swim pattern (such as time spent in the training quadrant and number of crossings exactly over the area that had contained the escape platform) were observed for a $40-\mathrm{sec}$ period. The average latencies for Trials 30-32, along with number of annulus crossings and time spent in the training quadrant, were used to identify cognitively impaired versus unimpaired aged rats. The rats were defined as impaired if they were more than 2 standard deviations below the mean for young rats in two out of three of the above measures. Using these criteria, $67 \%$ of the aged rats were considered impaired. This percentage is similar to what has been previously reported for 23-25-month-old Fischer 344 rats (Fischer, Bjorklund, Chen, \& Gage, 1991; Fischer, Sirevaag, Wiegand, Lindsay, \& Bjorklund, 1994). All rats were also given eight cue learning trials prior to treatment in order to assess the ability of GDNF to improve noncognitive aspects of performance in the Morris maze. These cue learning trials were conducted prior to initial place training, with the escape platform in the same position for both versions of the task.

Two weeks after the first training phase, all rats were given bolus injections of either GDNF (100 $\mu \mathrm{g} / 5 \mu \mathrm{l})$ or a phosphate-buffered saline (PBS) vehicle into the lateral ventricle. Bolus injections were chosen for two reasons: (1) a single injection might be more desirable as a clinical application, and (2) earlier work had shown that when GDNF was given as a $10-\mu \mathrm{g}$ bolus, it partially normalized age-related motor and neurochemical deficits (Bowenkamp, Lapchak, Hoffer, \& Bickford, 1996; Hebert \& Gerhardt, 1997). Unfortunately for the purposes of this study, the only data set showing that GDNF could alter hippocampal cholinergic measures in aged rats used a continuous infusion, noting efficacy at 7 days to 2 weeks (Lapchak et al., 1997). The dose used in the above study was $10 \mu \mathrm{g} /$ day. Therefore, we calculated our dose for a single injection on the basis of the total received during the continuous infusion $(10 \mu \mathrm{g} \times 14$ days $=140 \mu \mathrm{g})$ and the limits of solubility, which gave us the $100-\mu \mathrm{g}$ bolus dose.

Recombinant human GDNF was made, purified, and diluted as described by Williams et al. (1996). Injections were done under isoflurane anesthesia, using the following sterotaxic coordinates: (flat skull) anterior/posterior, $-0.8 \mathrm{~mm}$; medial/lateral, $1.2 \mathrm{~mm}$; and dorsal $/$ ventral, $-3.5 \mathrm{~mm}$ (young rats) and $-4.0 \mathrm{~mm}$ (aged rats) from top of skull. The dorsal/ventral coordinates were deeper in the aged rats than in the young rats because of differences in skull thickness. Six days after injection, all rats continued place training with the hidden platform in the same location as during the first phase of training. The time frame for continuation of water maze testing was based on the Lapchak et al. (1997) study, in which aged rats showed elevations in locomotor activity 7 days after infusion onset and showed elevations in striatal and septal ChAT activity 2 weeks after infusion onset.

During this second phase of training, the rats were again given four trials each day, with a probe trial inserted every eighth trial. All rats were trained in this way until they met a spatial learning criterion that was based on performance during the probe trials. The rats met criterion if they spent at least $14 \mathrm{sec}$ in the training quadrant and if they made at least two annulus crossings. These criteria were based on previous work in which a similar proportion of time spent in the training quadrant represented assymptotic performance in probe trials (Decker, Pelleymounter, \& Gallagher, 1988). Upon meeting these criteria, the rats were given eight more cue training trials, with the cue platform in a location that differed from the hidden platform. The cue platform was moved to reduce the possibility that the aged rats would use memorized, spatial strategies to locate the cue platform and would therefore not control for any GDNFinduced changes in nonspatial learning. The rats were then sacrificed to assess the accuracy of the cannula implant and to look for any signs of pathology, such as pituitary tumors. Any rat with clear evidence of pathology was dropped from statistical analysis.

\section{Statistics}

Escape latencies were analyzed using a repeated measures analysis of variance (ANOVA; age group $\times$ treatment $\times$ day) in which the dependent variable was latency from day to day. Swim speed was analyzed the same way, but with trials averaged over 2 days, giving only seven samples of swim speed over the 14 days of treatment. Since the rats were dropped from the analysis when they reached criterion, ANOVAs were conducted only during time points where the groups being compared were still intact. Cue learning data were analyzed using a repeated measures ANOVA (age group $\times$ treatment $X$ trial) in which the dependent variable was latency from trial to trial. Trials to criteria were analyzed using a two-way ANOVA (age group $\times$ treatment), with Fischer's PLSD as the post hoc testing procedure when main effects in the ANOVA were significant.

\section{RESULTS}

Figure 1A shows that the aged impaired rats required significantly more probe trials to reach our spatial bias criterion than did their unimpaired counterparts $[F(2,50)=$ $47.7, p<.0001]$. GDNF significantly improved this measure of acquisition rate in the aged impaired rats $[F(2,50)=6.6, p<.003]$ (post hoc $p<.0005)$ but had no effect in the aged unimpaired rats $(p<.39)$ or their young counterparts $(p<1.0)$. The aged impaired rats also had higher overall escape latencies throughout both pretreatment $[F(2,350)=77.12, p<.0001]$ and the first 2 days of the posttreatment $[F(2,50)=66.5, p<.0001]$ test periods than did either the aged unimpaired rats ( $p s<.0008-.0001)$ or the young rats $(p s<.0001)$. During the first 2 days of post-GDNF training (prior to any treatment or age group reaching criteria), all groups showed improvement $[F(1,50)=18.9, p<.0001]$, but there were no GDNF-related effects. By the 5th postGDNF training day, however, escape latencies were significantly reduced in the aged impaired rats treated with GDNF relative to their counterparts treated with PBS $[F(1,30)=34.8, p<.0002]$. There were no significant group assignment effects for these aged impaired rats prior to treatment $(p<.195)$. All of the above latency data are illustrated in Figure 1B. There was a similar trend when total distance to find the platform was used as the dependent measure, with a significant interaction between treatment and trial $[F(1,20)=3.2, p<.02]$ when an ANOVA was conducted on data from the aged impaired rats on Days 3-8. When distance was divided by latency to derive a measure of swim speed, there were no significant treatment effects $[F(1,4)=0.23, p<.65]$ or interactions between treatment and trial $[F(1,4)=1.1$, $p<.35]$ during the same time period in the aged impaired rats. Data are not shown for either total distance or swim speed.

After treatment, cue learning significantly improved with time $[F(7,175)=13, p<.0001]$ for all rats, and the young rats were significantly faster at locating the visible 
A)

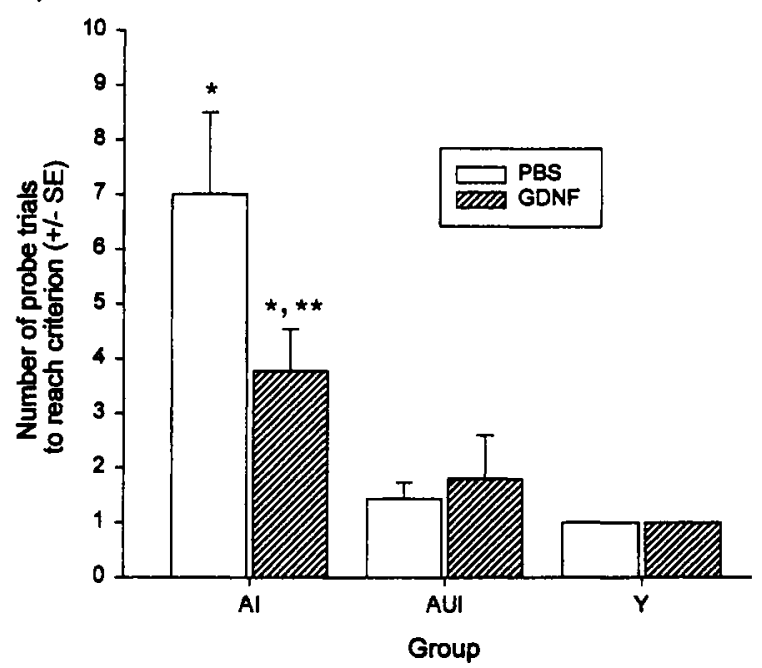

B)

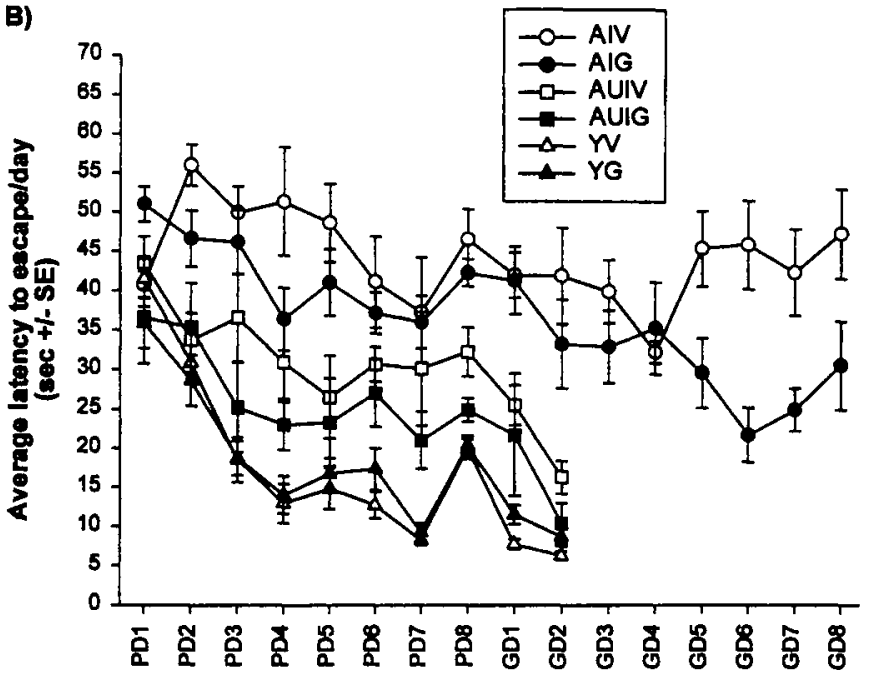

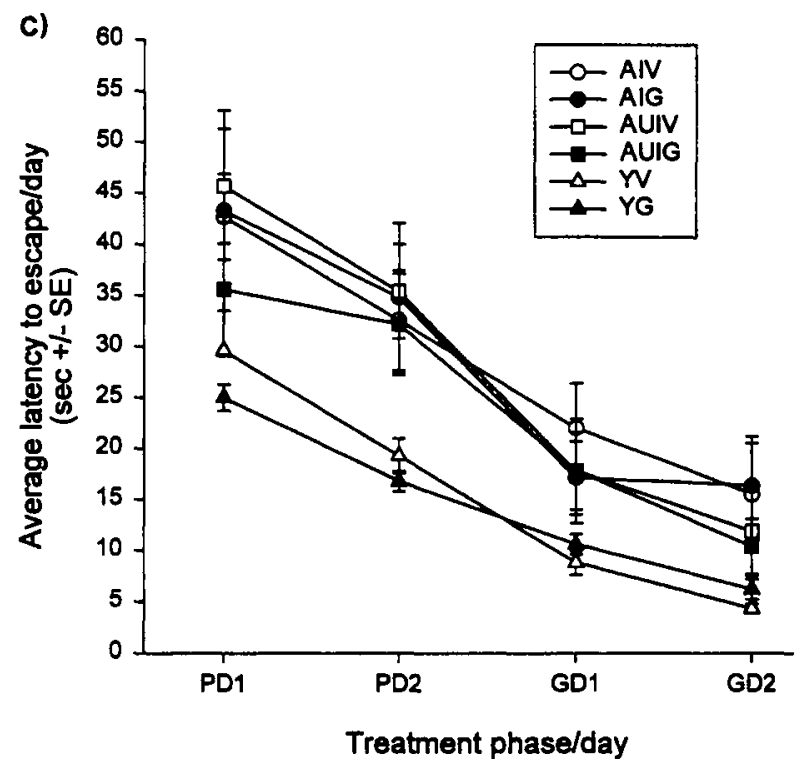

Figure 1. Effects of GDNF on spatial learning in aged impaired (AI), aged unimpaired (AUI), and young (Y) rats. (A) Average number of trials to criterion for AI, AUI, and Y rats treated with either PBS or GDNF (100- $\mu$ g icv bolus 6 days prior to the onset of testing). ${ }^{*} p<.0001$ versus AUI and Y. ${ }^{* *} p<.0005$ versus PBS of that group. (B) Average latency to escape onto the hidden platform averaged over days prior (PD) to GDNF treatment or after (GD) GDNF treatment for AI, AUI, and Y rats. Data are not shown for time points past GD8, when many of the rats had met criterion and were dropped from analysis. It should be noted that the rats began to meet criterion after GD2. (C) Average latency to escape onto the visible platform averaged over days prior (PD) to GDNF treatment or after (GD) GDNF treatment. The visible platform was moved to a different location for the post-GDNF treatment trials. For YV, YG, AUIV, AUIG, AIV, and AIG, $n s=15,15,7,5,5$, and 9, respectively.

platform than were the aged rats $[F(2,175)=10.5, p<$ $.0005]$. The same pattern was observed for the pretreatment location, with significant improvement over time $[F(7,175)=15.8, p<.0001]$ as a main effect and the young rats showing much faster escape latencies overall $[F(2,175)=17.8, p<.0001]$. Furthermore, cue learning escape latencies were more rapid during the second cue training session, despite the different location, than during the first $[F(15,375)=24.2, p<.0001]$ for all groups.
GDNF did not significantly affect either the absolute cue learning latencies or the pretraining versus posttraining improvement in cue training latencies in any age group. Cue latencies are shown in Figure 1C.

\section{DISCUSSION}

GDNF significantly improved the acquisition rate of the aged rats for the Morris maze task, regardless of the 
measure used to assess acquisition. It reduced the number of probe trials to reach criterion, which is more dependent on search accuracy than other measures, such as escape latency. GDNF also reduced escape latencies and swim distances over time, doubling the slope of the curve generated by plotting trial day against latency for the aged impaired rats. These effects of GDNF appear to reflect a true improvement in spatial learning for the following reasons: (1) accuracy measures, such as probe trials to criterion, were improved along with measures that could reflect nonspatial aspects of performance (e.g., escape latency), (2) swim distance was reduced with time, and (3) nonspatial aspects of performance, such as swim speed and acquisition of the cued version of the task, were not affected by GDNF.

Exactly how GDNF improved spatial learning cannot be determined from this preliminary data set. Reduced septal or stratal ChAT has been correlated with a spatial learning impairment in aged rats (Gallagher et al., 1990). Since GDNF has been shown to increase septohippocampal ChAT activity in aged rats (Lapchak et al., 1997), it is possible that GDNF improved spatial learning in the aged rats by increasing or nomalizing septohippocampal cholinergic activity. Cholinergic deficits may not be the primary basis of the spatial learning impairment observed in the aged rats, however, since septal cholinergic lesions do not induce spatial learning impairments in aged rats (Baxter \& Gallagher, 1996). Furthermore, changes in a number of other transmitter systems, including hippocampal and cortical glutamate, dopamine, and serotonin, have also been associated with age-related spatial learning impairments (Gallagher et al., 1990; Lee et al., 1994). All of the above suggests that age-related spatial learning impairments probably reflect changes in multiple biological systems.

GDNF, then, could improve spatial learning by interacting with any one of the above systems in aged rats. Indeed, GDNF receptor alpha has been localized in the cortex, hippocampus, and septum (Araujo et al., 1997; Nosrat, Tomac, Hoffer, \& Olson, 1997), and could therefore affect the synaptic strength of contacts between any neurotransmitter systems found in the above brain areas. In addition, the GDNF signaling protein, c-RET tyrosine kinase, is expressed in the septohippocampal pathway and is reduced in the septum after fimbria-fornix transection (Araujo et al., 1997). Although it is not clear whether GDNF or its receptor/signaling protein complex is similarly regulated in aged animals, it is possible that GDNF could act to partially restore synaptic plasticity to compromised cortical or septohippocampal neurons, regardless of transmitter phenotype.

In summary, GDNF improved spatial learning in aged Fisher 344 rats, in that impaired aged rats treated with GDNF showed a significant reduction in escape latencies and in the number of probe trials to criterion relative to their PBS-treated impaired counterparts. GDNF did not alter nonspatial aspects of water maze learning, such as cue learning and swim speed. These data support the idea that (1) GDNF can support the integrity of neural circuitry which is compromised by the aging process and (2) GDNF might be effective in systems that are not primarily associated with motor function, such as the septum and hippocampus.

\section{REFERENCES}

Araujo, D. M., Hilt, D., Miller, P. J., Wen, D., Jiao, S., \& Lapchak, P. A. (1997). RET receptor tyrosine kinase immunoreactivity is altered in glial cell line-derived neurotrophic factor-responsive neurons following lesions of the nigrostriatal and septohippocampal pathways. Neuroscience, 80, 9-16.

BAXTER, M. G., \& GallaGHer, M. (1996). Intact spatial learning in both young and aged rats following selective removal of hippocampal cholinergic input. Behavioral Neuroscience, 110, 460-467.

Beck, K. D., Valverde, J., Alexi, T., Poulsen, K., Moffat, B., VandLen, R. A., Rosenthal, A., \& Hefti, F. (1995). Mesencephalic dopaminergic neurons protected by GDNF from axotomy-induced degeneration in the adult rat brain. Nature, 373, 339-341.

Bowenkamp, K. E., Lapchak, P. A., Hoffer, B. J., \& Bickford, P. C. (1996). Glial cell line-derived neurotrophic factor reverses motor impairment in 16-17 month-old rats. Neuroscience Letters, 211, 81-84.

Decker, M. W., Pelleymounter, M. A., \& Gallagher, M. (1988), Effects of training on a spatial memory task on high affinity choline uptake in hippocampus and cortex in young adult and aged rats. Journal of Neuroscience, 8, 90-99.

Devan, B. D., Goad, E. H., \& Petri, H. L. (1996). Dissociation of hippocampal and striatal contributions to spatial navigation in the water maze. Neurobiology of Learning \& Memory, 66, 305-323.

Eichenbaum, H., STEwarT, C., \& MorRIs, R. G. (1990). Hippocampal representation in place learning. Journal of Neuroscience, 10, 35313542.

Fischer, W., BJoRkLUnd, A., CHEN, K. S., \& GAGE, F. H. (1991). NGF improves spatial memory in aged rodents as a function of age. Journal of Neuroscience, 11, 1889-1906.

Fischer, W., SirevaAg, A., Wiegand, S. J., Lindsay, R. M., \& BJorKLUND, A. (1994). Reversal of spatial memory impairments in aged rats by nerve growth factor and neurotrophins 3 and $4 / 5$ but not by brain-derived neurotrophic factor. Proceedings of the National Academy of Science, 91, 8607-8611.

Gallagher, M., Burwell, R. D., Kodsi, M. H., MCKinney, M., Southerland, S., Vella-RountreE, L., \& Lewis, M. H. (1990). Markers for biogenic amines in the aged rat brain: Relationship to decline in spatial learning ability. Neurobiology of Aging, 11, 507. 514.

Hebert, M. A., \& Gerhardt, G. A. (1997). Behavioral and neurochemical effects of intranigral administration of glial cell linederived neurotrophic factor on aged Fischer 344 rats. Journal of Pharmacology \& Experimental Therapeutics, 282, 760-768.

Henderson, C. E., Phillips, H. S., Pollock, R. A., Davies, A. M., Lemeulle, C., Armanini, M., Simpson, L., Moffet, B., Vandlen, R. A., Koliatsos, V., \& Rosenthal, A. (1994). GDNF: A potent survival factor for motoneurons present in peripheral nerve and muscle. Science, 266, 1062-1064

Hoffer, B. J., Hoffman, A., Bowenkamp, K., Huettl, P., Hudsonk, J., Martin, D., Lin, L.-F., \& Gerhardt, G. A. (1994). Glial cell linederived neurotrophic factor reverses toxin-induced injury to midbrain dopaminergic neurons in vivo. Neuroscience Letters, 182, 107-111.

KeARNS, C. M., \& GASH, D. M. (1995). GDNF protects nigral dopamine neurons against 6-hydroxydopamine in vivo. Brain Research, 672, 104-111.

LaPChaK, P. A., Miller, P. J., \& JiaO, S. (1997). Glial cell line-derived neurotrophic factor induces the dopaminergic and cholinergic phenotype and increases locomotor activity in aged Fischer 344 rats. Neuroscience, 77, 745-752.

Lee, J. M., Ross, E. R., Gower, A., Paris, J. M., Martensson, R., \& LORENS, S. A. (1994). Spatial learning deficits in the aged rat: Neuroanatomical and neurochemical correlates. Brain Research Bulletin, 33, 489-500.

Lin, L.-F., Doherty, D. H., Lile, J. D., Bektesh, S., \& Collins, F. 
(1993). GDNF: A glial cell-line derived neurotrophic factor for midbrain dopaminergic neurons. Science, 260, 1130-1132.

Nosrat, C. A., Tomac, A., Hoffer, B. J., \& Olson, L. (1997). Cellular and developmental patterns of expression of RET and glial cell linederived neurotrophic factor receptor alpha mRNAs. Experimental Brain Research, 115, 410-422.

OPPENheim, R. W., Houenou, L. J., JohnSON, J. E., LiN, L.-F., LI, L., Lo, A. C., Newsome, A. L., Prevette, D. M., \& Wang, S. (1995). Developing motor neurons rescued from programmed and axotomyinduced cell death by GDNF. Nature, 373, 344-346.

Sauer, H., Rosenblad, C., \& Buorklund, A. (1995). GDNF but not TGF-b3 prevents delayed degeneration of nigral dopaminergic neurons following striatal 6-hydroxydopamine lesion. Proceedings of the National Academy of Science, 92, 8935-8939.

Tomac, A., LindQuist, E., Lin, L. F., Ogren, S. O., Young, D., Hof-
FER, B. J., \& OLSON, L. (1995). Protection and repair of the nigrostriatal dopaminergic system by GNDF in vivo. Nature, 373, 335-339.

Williams, L. R., Inouye, G., Cummins, V., \& Pelleymounter, M. A. (1996). Glial cell-line-derived neurotrophic factor sustains axotomized basal forebrain cholinergic neurons in vivo: Dose response comparison to nerve growth factor and brain-derived neurotrophic factor. Journal of Pharmacology \& Experimental Therapeutics, 277, $1140-1151$

Yan, Q., Matheson, C., \& Lopez, O. T. (1995). In vivo neurotrophic effects of GDNF on neonatal and adult facial motor neurons. Nature, 373, 341-344.

(Manuscript received October 6, 1998; revision accepted for publication December $21,1998$. 